\title{
Circadian Rhythm and CKD: Is Melatonin a Key Player or Bi-player?
}

\author{
Yasuyuki Nagasawa, Yukiko Hasuike, Takahiro Kuragano and Masaharu Ishihara
}

Key words: melatonin, circadian rhythm, proteinuria, chronic kidney disease, sleep, sodium

(Intern Med 58: 1531-1532, 2019)

(DOI: 10.2169/internalmedicine.2323-18)

\section{Circadian Rhythm and Chronic Kidney Disease (CKD)}

The circadian rhythm is well known to have great influence upon many systemic mechanisms including the kidney function, blood pressure, salt intake and excretion, but not limited to sleep rhythm. In dipper-type patients, whose nighttime blood pressure is usually lower than their daytime blood pressure, salt intake sometimes attenuates the circadian rhythm of their blood pressure changes. Proteinuria, one of most important factors in CKD, is also reported to have a circadian rhythm (Fig. 1A) (1). The level of proteinuria, especially from 03:00 to 06:00, was found to be clearly lower than that during the daytime. Originally, this circadian rhythm of proteinuria was considered to be caused by meal and salt intake during daytime (1). Recently, proteinuria was recognized as the balance of the excretion of proteinuria in the glomeruli and the re-absorption of proteinuria in the renal tubuli (2). The circadian rhythm of proteinuria might also be caused by this balance, because the renal tubular function is altered by various hormones that have circadian rhythm. Recently, many genes related to circadian rhythm have been reported to be expressed in the kidneys $(3,4)$, which might also contribute to the circadian rhythm of proteinuria.

\section{Circadian Rhythm and Melatonin}

Melatonin is an important circadian rhythm-related hormone that is regulated by the pineal body under light exposure (5). Melatonin is elevated during nighttime, which causes sleepiness two hours after its peak elevation (Fig. 1B) (5, 6). Originally, role of melatonin was thought to be limited to the sleep rhythm. Recently, however, melatonin has been reported to be involved in autophagy, apopto-
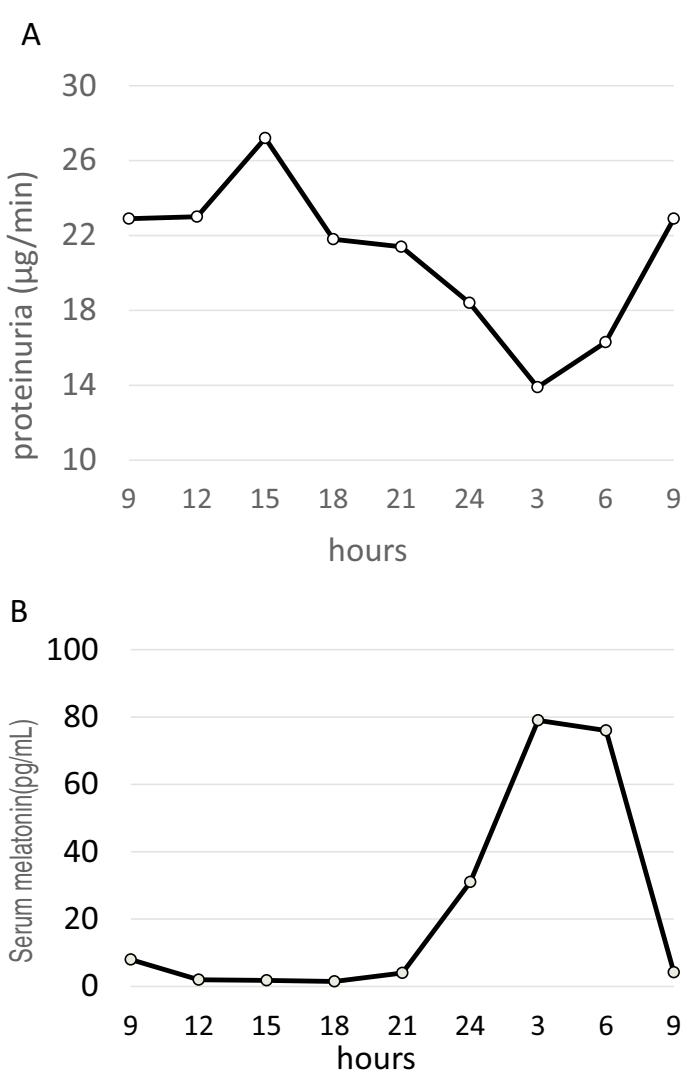

Figure 1. A: The circadian rhythm of proteinuria levels. Proteinuria has circadian rhythm. The proteinuria levels during nighttime, from 0:00 to 06:00 were lower than those during daytime. The figure was created from data reported by Buzio et al. (1). B: The circadian rhythm of melatonin levels. The melatonin level has a circadian rhythm. The melatonin levels during nighttime, from 0:00 to 06:00, were higher than those during daytime. The figure was adapted from the figure reported by Zhdanova et al. (6). 


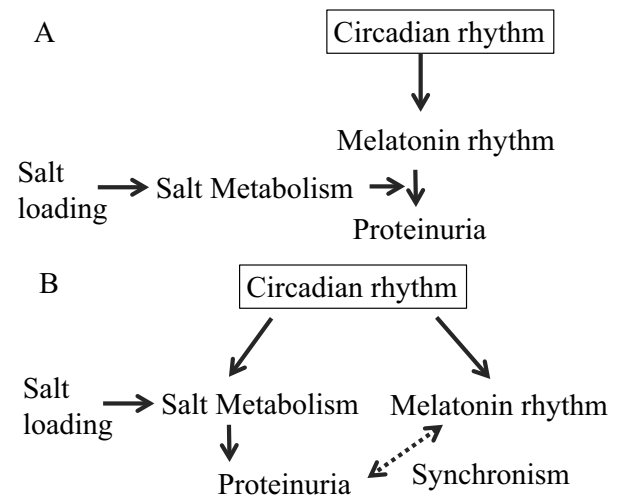

Figure 2. A: Possible mechanism of the relationship between melatonin and proteinuria if melatonin directly influences proteinuria. Melatonin has a circadian rhythm, resulting in the circadian rhythm of proteinuria. Salt loading might directly alter this relationship. B: Possible mechanism of the relationship between melatonin and proteinuria if melatonin does not directly influence proteinuria. Both of proteinuria and melatonin have circadian rhythm, resulting in the synchronism of these two parameters. Salt loading might independently alter proteinuria because a high salt intake usually increases proteinuria.

sis, liver metabolism, and cancer (5, 7-9), suggesting that melatonin is a multi-functional hormone. Interestingly, the time course of melatonin was found to have an exact mirror image of the time course of proteinuria (Fig. 1A). At 0:00 to 06:00, when melatonin was elevated, proteinuria was suppressed. The similarity of these time courses might indicate some relationship between melatonin and proteinuria. However, at this point, no direct relationship between melatonin and the kidney function has been reported.

\section{Salt Loading Might Alter the Relationship between Melatonin and Proteinuria}

In this current issue, Ohashi et al reported that the urinary melatonin level and proteinuria were negatively correlated during both daytime and nighttime under standard salt conditions, but that this correlation could not be observed under low salt conditions (10). The authors proposed the hypothesis that melatonin had some influence on proteinuria, and that salt loading attenuated this correlation (Fig. 2A). In this hypothesis, melatonin could account for the proteinuria in short sleepers (11), because short sleep should reduce melatonin levels. However, the levels of both melatonin and proteinuria have a circadian rhythm. Recently, genes related to circadian rhythm have been reported to enhance the systemic $\mathrm{Na}+/ \mathrm{K}+\mathrm{ATPase}$ beta subunit, resulting in the circadian rhythm of blood pressure (12). Moreover, salt metabolism shows some circadian rhythm, and salt loading itself has a great influence on proteinuria. Thus, there is another possi- ble mechanism wherein melatonin and proteinuria might be synchronic under the central circadian rhythm, with salt loading altering the proteinuria level independently (Fig. 2B). More direct evidence of the relationship between melatonin and proteinuria is expected, because melatonin can be used in the clinical settings and because melatonin might represent a new therapeutic target for proteinuria.

\section{Author's disclosure of potential Conflicts of Interest (COI).}

Yasuyuki Nagasawa: Honoraria, Japan Boehringer-Ingelheim, Japan Ily-Lilly and Otsuka Pharmaceutical. Takahiro Kuragano: Honoraria, Chugai Pharmaceutical, Kyowa Kirin and Bayer Yakuhin. Masaharu Ishihara: Honoraria, MSD, Bayer Yakuhin, Astellas Pharmaceutical, Daiichi Sankyo, SANOFI, Astra Zeneca, Takeda Pharmaceutical, Kissei Pharmaceutical and Shionogi; Research funding, Amgen Astellas Bio Pharma.

\section{References}

1. Buzio C, Mutti A, Capani F, et al. Circadian rhythm of proteinuria: effects of an evening meat meal. Nephrol Dial Transplant 4: 266-270, 1989

2. Wuerzner G, Firsov D, Bonny O. Circadian glomerular function: from physiology to molecular and therapeutical aspects. Nephrol Dial Transplant 29: 1475-1480, 2014.

3. Yamato M, Ito T, Iwatani H, Yamato M, Imai E, Rakugi H. Ecadherin and claudin-4 expression has circadian rhythm in adult rat kidney. J Nephrol 23: 102-110, 2010.

4. Firsov D, Bonny O. Circadian rhythms and the kidney. Nat Rev Nephrol 14: 626-635, 2018.

5. Zisapel N. New perspectives on the role of melatonin in human sleep, circadian rhythms and their regulation. Br J Pharmacol 2018 (Epub ahead of print).

6. Zhdanova IV, Wurtman RJ, Balcioglu A, Kartashov AI, Lynch HJ. Endogenous melatonin levels and the fate of exogenous melatonin: age effects. J Gerontol A Biol Sci Med Sci 53: B293-B298, 1998.

7. Roohbakhsh A, Shamsizadeh A, Hayes AW, Reiter RJ, Karimi G. Melatonin as an endogenous regulator of diseases: the role of autophagy. Pharmacol Res 133: 265-276, 2018.

8. Farhood B, Goradel NH, Mortezaee K, Khanlarkhani N, Najafi M, Sahebkar A. Melatonin and cancer: from the promotion of genomic stability to use in cancer treatment. J Cell Physiol 2018 (Epub ahead of print).

9. Luo C, Yang Q, Liu Y, et al. The multiple protective roles and molecular mechanisms of melatonin and its precursor $\mathrm{N}$ acetylserotonin, in targeting brain injury and liver damage and in maintaining bone health. Free Radic Biol Med 130: 215-233, 2018.

10. Ohashi N, Ishigaki $S$, Isobe $S$, et al. Salt loading aggravates the relatiohship between melatonin and proteinuria in patients with chronic kidney disease. Intern Med 58: 1557-1564, 2019.

11. Yamamoto R, Nagasawa Y, Iwatani H, et al. Self-reported sleep duration and prediction of proteinuria: a retrospective cohort study. Am J Kidney Dis 59: 343-355, 2012.

12. Nakashima A, Kawamoto T, Noshiro M, et al. Dec1 and CLOCK regulate $\mathrm{Na}^{+} / \mathrm{K}^{+}$-ATPase $\beta 1$ subunit expression and blood pressure. Hypertension 72: 746-754, 2018.

The Internal Medicine is an Open Access journal distributed under the Creative Commons Attribution-NonCommercial-NoDerivatives 4.0 International License. To view the details of this license, please visit (https://creativecommons.org/licenses/ by-nc-nd/4.0/).

\footnotetext{
(C) 2019 The Japanese Society of Internal Medicine
} Intern Med 58: 1531-1532, 2019 